Supporting Information

\title{
Mesoporous Silica Thin Membrane with Tunable Pore Size for Ultrahigh Permeation and Precise Molecular Separation
}

Jingling Yang, ${ }^{+a, b}$ Geng-Sheng Lin, ${ }^{+c}$ Chung-Yuan Mou, ${ }^{{ }_{a}}$ and Kuo-Lun Tung ${ }^{* c, d}$

a. Department of Chemistry, National Taiwan University, No. 1, Sec. 4 Roosevelt Rd. Taipei 10617, Taiwan.

b. School of Environment, Jinan University, Guangzhou 510632, China.

c. Department of Chemical Engineering, National Taiwan University, No. 1, Sec. 4 Roosevelt Rd. Taipei 10617, Taiwan.

d. Advanced Research Center for Green Materials Science and Technology, National Taiwan University, No. 1, Sec. 4 Roosevelt Rd. Taipei 10617, Taiwan.

* Corresponding author: cymou@ntu.edu.tw; kltung@ntu.edu.tw

+ These authors contributed equally to this work 


\section{Experimental Section}

\section{Synthesis of a biomimetic MSTF $\perp$ AAO membrane by the polymer interlayer method.}

First, polystyrene (PS) was used to coat a smooth surface layer on the macropores of anodic aluminum (AAO) oxide membranes for subsequent growth of a mesoporous silica thin film (MSTF). PS ( $2.0 \mathrm{~g}$, MW 260,000, ACROS) was dissolved in $20.0 \mathrm{~g}$ toluene at $50^{\circ} \mathrm{C}$ for $1 \mathrm{~h}$, and then $0.25 \mathrm{wt} \%$ photo-initiator (2-hydroxy-4-(2-hydroxyethoxy)-2-methylpropiophenone) was dissolved in the above solutions. Then, 700 $\mu \mathrm{L}$ mixed PS solutions were used to spin-coat the AAO membranes $\left(4.7 \mathrm{~cm}\right.$ in diameter, , pore size of $147 \mathrm{~nm}$, Whatman ${ }^{\oplus}$, situated on a $5 \times 5$ $\mathrm{cm}^{2}$ glass sheet) at $2000 \mathrm{rpm}$ for $30 \mathrm{~s}$, and then immediate cross-linking of the polymers on the AAO surface was performed under UV light irradiation for $5 \mathrm{~min}$. The spin-coating and cross-linking procedures were repeated three times. Subsequent curing of the PS on AAO supports at $100{ }^{\circ} \mathrm{C}$ for $1 \mathrm{~h}$ resulted in the as-prepared membrane labelled PS/AAO. Then, a single-layer MSTF was grown on the top surface of the PS/AAO membrane in an oil-in-water emulsion. The oil-in-water emulsion was prepared by mixing cetyltrimethylammonium bromide $(\mathrm{CTAB})(0.965 \mathrm{~g})$, ethanol $(30.0 \mathrm{~g})$ and oil pore-expanding agent $(0.015 \mathrm{~mol})$ at $50^{\circ} \mathrm{C}$. Then, the PS/AAO membrane was immersed in the microemulsion, followed by the introduction of $\mathrm{NH}_{3}$ aqueous solution $(7.5 \mathrm{~g}, 35.5 \mathrm{wt} \%)$ and tetraethyl orthosilicate (TEOS)/ethanol solution ( $8.35 \mathrm{~mL}, 20 \%$ by volumes) under stirring at $50^{\circ} \mathrm{C}$ for $1 \mathrm{~h}$. Then, the reaction was aged at $50^{\circ} \mathrm{C}$ for $20 \mathrm{~h}$ without stirring. The molar ratio of cetyltrimethylammonium bromide $(\mathrm{CTAB}) / \mathrm{H}_{2} \mathrm{O} / \mathrm{NH}_{3} /$ pore-expanding agent/ethanol/tetraethyl orthosilicate (TEOS) was set to 1:8400:90:5.8:250:2.8. ${ }^{1}$ The pore size of the MSTF was tuned via introducing different pore-expanding agents during synthesis. Thereafter, the as-synthesized membrane was rinsed with ethanol, calcined from 30 to $400^{\circ} \mathrm{C}$ with a ramp rate of $1{ }^{\circ} \mathrm{C} / \mathrm{min}$ in air and maintained at $400{ }^{\circ} \mathrm{C}$ for $30 \mathrm{~min}$, followed by UV ozone cleaning for $30 \mathrm{~min}$. The as-prepared MSTF $\perp$ AAO membranes were labeled P-MSTF $\perp$ AAO, D-MSTF $\perp \mathrm{AAO}, \mathrm{H}-\mathrm{MSTF} \perp \mathrm{AAO}$, and $\mathrm{E}-\mathrm{MSTF} \perp \mathrm{AAO}$, where $\mathrm{P}, \mathrm{D}, \mathrm{H}$ and $\mathrm{E}$ are abbreviations for the pore-expanding agents used: pentyl ether, decane, hexadecane, and ethyl acetate, respectively.

Table S1. Dye molecules and their properties. 


\begin{tabular}{lccllc}
\hline Dye & Charge & $\begin{array}{l}\text { MW } \\
(\mathrm{Da})\end{array}$ & Molecular size & $\begin{array}{l}\mathrm{pH} \\
\text { value }\end{array}$ & $\mathrm{pKa}$ \\
\hline Reactive red 120 & - & 1469.98 & $2.1 \mathrm{~nm} \times 1.4 \mathrm{~nm}$ & 6.1 & 2.1 \\
Evans blue & - & 960.80 & $3.1 \mathrm{~nm} \times 1.2 \mathrm{~nm}$ & 7.0 & $-1.4^{\mathrm{a}}$ \\
Rose bengal & - & 1017.60 & $1.5 \mathrm{~nm} \times 1.2 \mathrm{~nm}$ & 5.9 & 4.7 \\
Congo red & - & 696.68 & $2.5 \mathrm{~nm} \times 0.7 \mathrm{~nm}$ & 6.4 & 4.1 \\
Rhodamine B & + & 479.02 & $1.2 \mathrm{~nm} \times 1.1 \mathrm{~nm}$ & 3.6 & 3.7 \\
Methylene blue & + & 319.85 & $1.3 \mathrm{~nm} \times 0.5 \mathrm{~nm}$ & 3.0 & 3.8 \\
\hline
\end{tabular}

a. $\mathrm{pKa}_{1}$ for Evans blue. 
Table S2. Dye/salt separation performance of nanofiltration membranes in literatures and in this work.

\begin{tabular}{|c|c|c|c|c|c|}
\hline Membrane & Dye & $\begin{array}{l}\text { Dye } \\
\text { Rejection } \\
(\%)\end{array}$ & $\begin{array}{l}\text { PWP } \\
\text { (LMH/bar) }\end{array}$ & Salt rejection (\%) & Ref. \\
\hline \multirow[t]{2}{*}{$\mathrm{CMCNa} / \mathrm{PP} \mathrm{NF}$} & Methyl blue (779.8 Da) & 99.6 & \multirow[t]{2}{*}{10.8} & $\mathrm{NaCl}: 28.8$ & \multirow[t]{2}{*}{2} \\
\hline & Congo red $(696.7 \mathrm{Da})$ & 99.8 & & $\mathrm{Na}_{2} \mathrm{SO}_{4}: 85.5$ & \\
\hline \multirow{2}{*}{$\begin{array}{l}\text { Polypiperazine- } \\
\text { amide NF }\end{array}$} & \multirow[t]{2}{*}{ Reactive black 5 (991.8 Da) } & \multirow[t]{2}{*}{99.3} & \multirow[t]{2}{*}{$\sim 7.0$} & $\mathrm{NaCl}: 66.4$ & \multirow[t]{2}{*}{3} \\
\hline & & & & $\mathrm{Na}_{2} \mathrm{SO}_{4}: 98.5$ & \\
\hline $\begin{array}{l}\text { Polyvinylamine } \\
\text { TMC NF }\end{array}$ & Methyl blue (779.8 Da) & 98.9 & 8.5 & $\mathrm{NaCl}: 61.6$ & 4 \\
\hline \multirow[t]{2}{*}{$\mathrm{mHT} / \mathrm{PES}$} & Reactive black 5(991.8 Da) & 95.0 & \multirow[t]{2}{*}{6.3} & \multirow[t]{2}{*}{$\mathrm{NaCl}: \sim 8.0$} & \multirow[t]{2}{*}{5} \\
\hline & Reactive red 49 (576.5 Da) & 90.0 & & & \\
\hline \multirow[t]{2}{*}{ Sepro NF 2A } & Congo red $(696.7 \mathrm{Da})$ & $\sim 100$ & \multirow[t]{2}{*}{10.5} & \multirow[t]{2}{*}{$\mathrm{NaCl}: 25.9$} & \multirow[t]{4}{*}{6} \\
\hline & Direct red $23(813.7 \mathrm{Da})$ & $\sim 100$ & & & \\
\hline \multirow[t]{2}{*}{ Sepro NF 6} & Congo red $(696.7 \mathrm{Da})$ & 99.9 & \multirow[t]{2}{*}{13.7} & \multirow[t]{2}{*}{$\mathrm{NaCl}: 10.7$} & \\
\hline & Direct red $23 \quad(813.7 \mathrm{Da})$ & 99.8 & & & \\
\hline \multirow[t]{2}{*}{ GO-PSBMA/PES } & Reactive black $5 \quad$ (991.8 Da) & 99.2 & \multirow[t]{2}{*}{$\sim 11.98$} & $\mathrm{NaCl}: \sim 4.0$ & \multirow[t]{2}{*}{7} \\
\hline & Reactive red 49 (576.5 Da) & 97.2 & & $\mathrm{Na}_{2} \mathrm{SO}_{4}: \sim 10.0$ & \\
\hline \multirow[t]{3}{*}{ Co-NF-2 } & Direct red $23 \quad(813.7 \mathrm{Da})$ & 99.5 & \multirow[t]{3}{*}{$\sim 18.2$} & $\mathrm{NaCl}: 3.3$ & \multirow[t]{3}{*}{8} \\
\hline & Congo red $(696.7 \mathrm{Da})$ & 99.4 & & & \\
\hline & Reactive blue 2 (774.2 Da) & 99.0 & & $\mathrm{Na}_{2} \mathrm{SO}_{4}: 25.2$ & \\
\hline \multirow[t]{4}{*}{ GR82PP } & Direct red $80(1373.07 \mathrm{Da})$ & 99.9 & \multirow[t]{4}{*}{37.5} & \multirow[t]{2}{*}{$\mathrm{NaCl}: \sim 0.35$} & \multirow[t]{4}{*}{9} \\
\hline & Direct red $23(813.7 \mathrm{Da})$ & 99.2 & & & \\
\hline & Reactive blue 2 (774.2 Da) & 99.9 & & $\mathrm{Na}_{2} \mathrm{SO}_{4}: \sim 3$ & \\
\hline & Reactive orange $16(617.54 \mathrm{Da})$ & 92.0 & & & \\
\hline \multirow[t]{2}{*}{$\mathrm{E}-\mathrm{MSTF}(+) \mathrm{AAO}$} & Evans blue (960.8 Da) & 99.0 & \multirow[t]{2}{*}{$\sim 270$} & $\mathrm{NaCl}: 3$ & \multirow[t]{2}{*}{$\begin{array}{l}\text { This } \\
\text { work }\end{array}$} \\
\hline & Evans blue $(960.8 \mathrm{Da})$ & 99.0 & & $\mathrm{Na}_{2} \mathrm{SO}_{4}: 4$ & \\
\hline
\end{tabular}



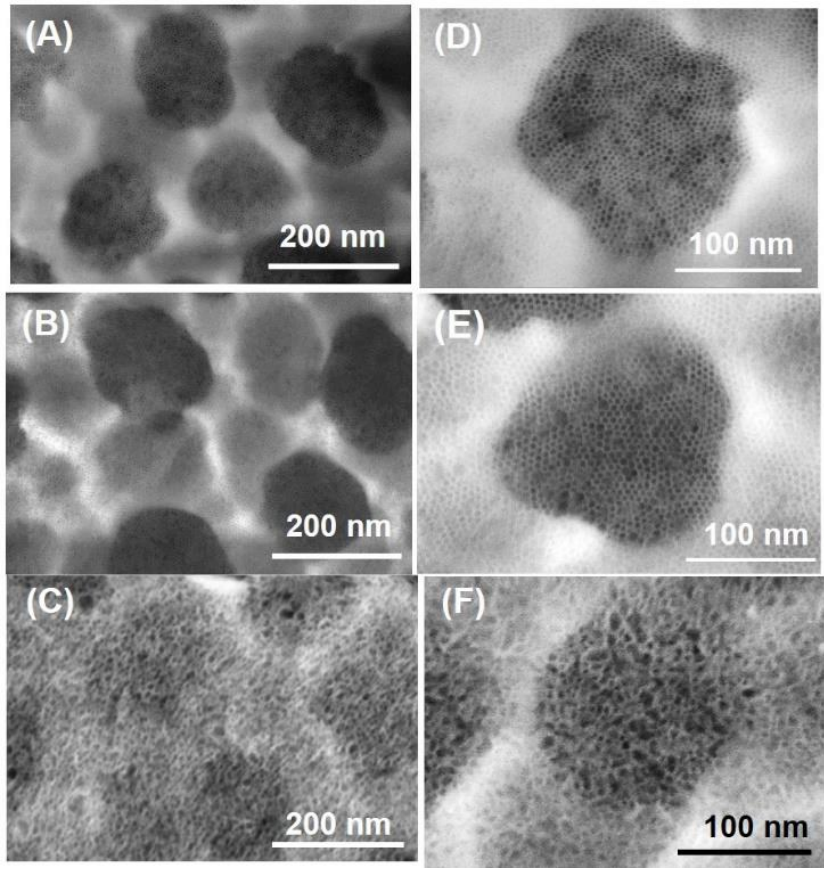

Figure S1. Low-magnification top-view SEM images of (A) H-MSTF $\perp$ AAO, (B) D-MSTF $\perp A A O$, and (C) $\mathrm{P}-\mathrm{MSTF} \perp \mathrm{AAO}$, highmagnification SEM images of (D) H-MSTF $\perp A A O$, (E) D-MSTF $\perp A A O$, and (F) P-MSTF $\perp A A O$.

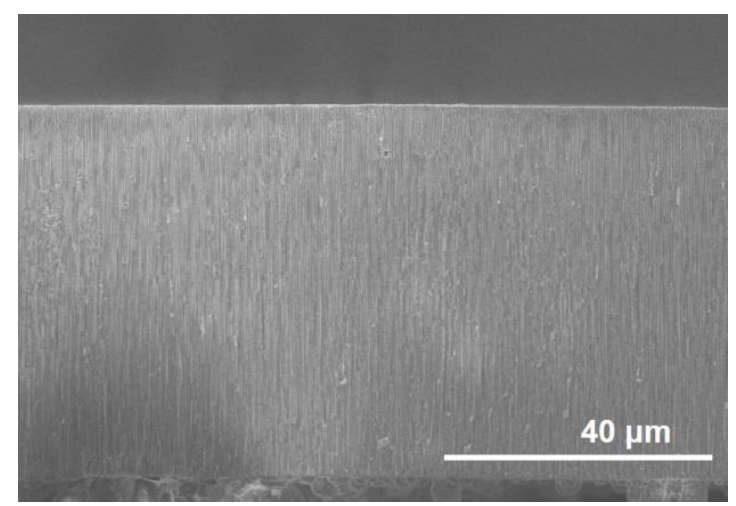

Figure S2. Cross-sectional SEM image of E-MSTF $\perp$ AAO. 


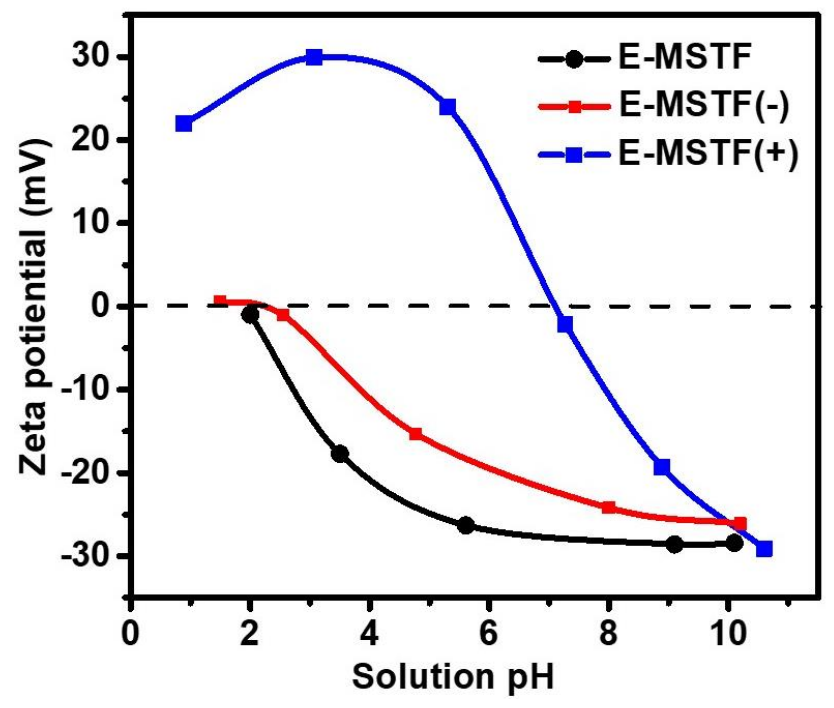

Figure S3. Zeta potentials of E-MSTF, E-MSTF(-), and E-MSTF(+).

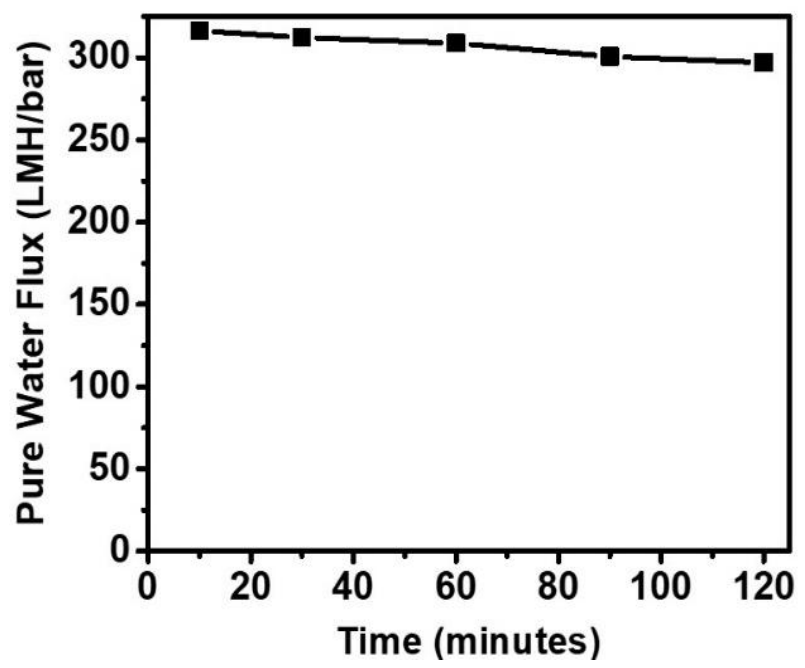

Figure S4. Time profile of pure water flux using the E-MSTF (+) membrane. 

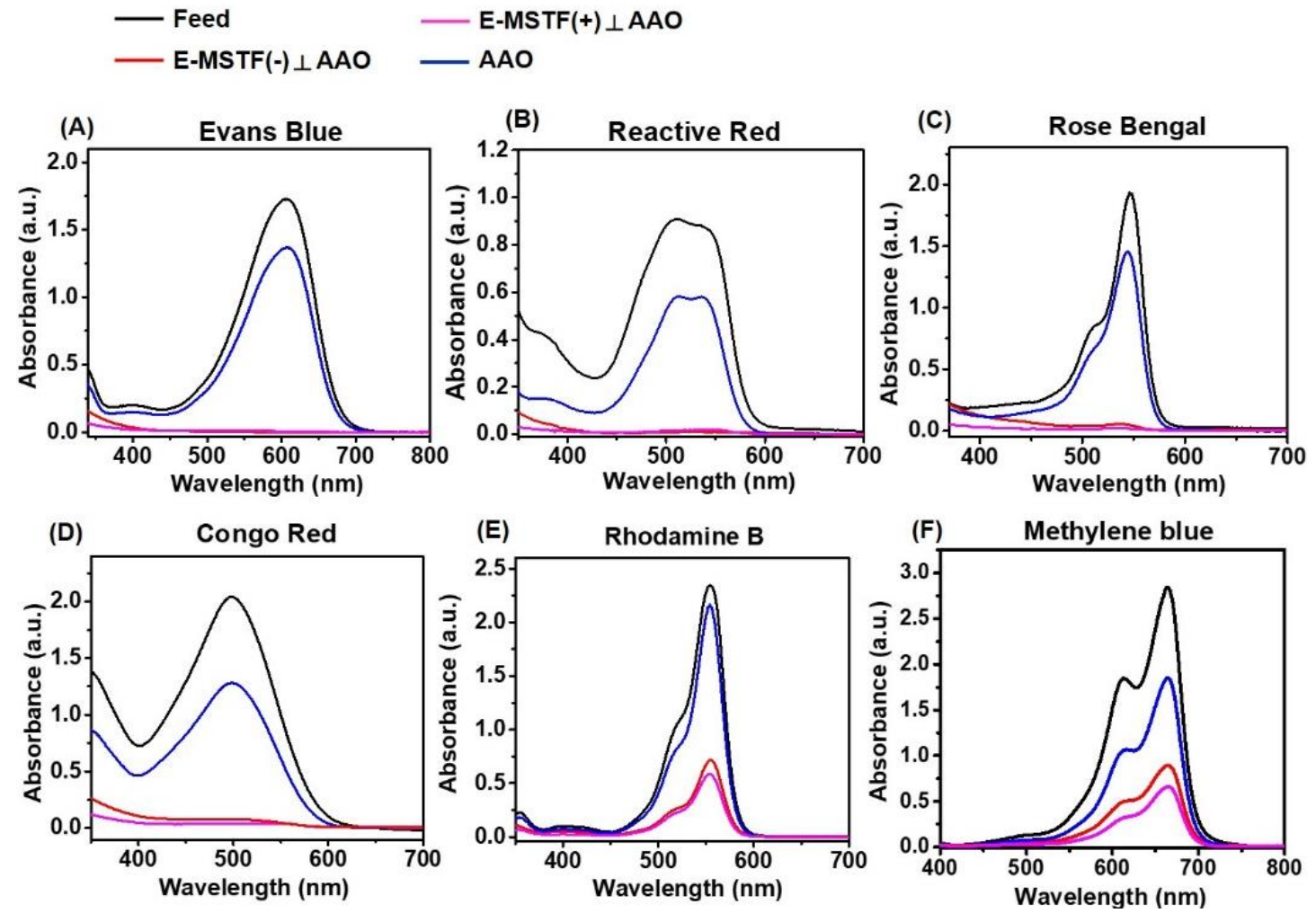

Figure S5. UV-vis absorption spectra of solutions of (A) EB, (B) RR, (C) RB, (D) CR, (E) RhB, and (F) MB before and after filtration through the filter device.
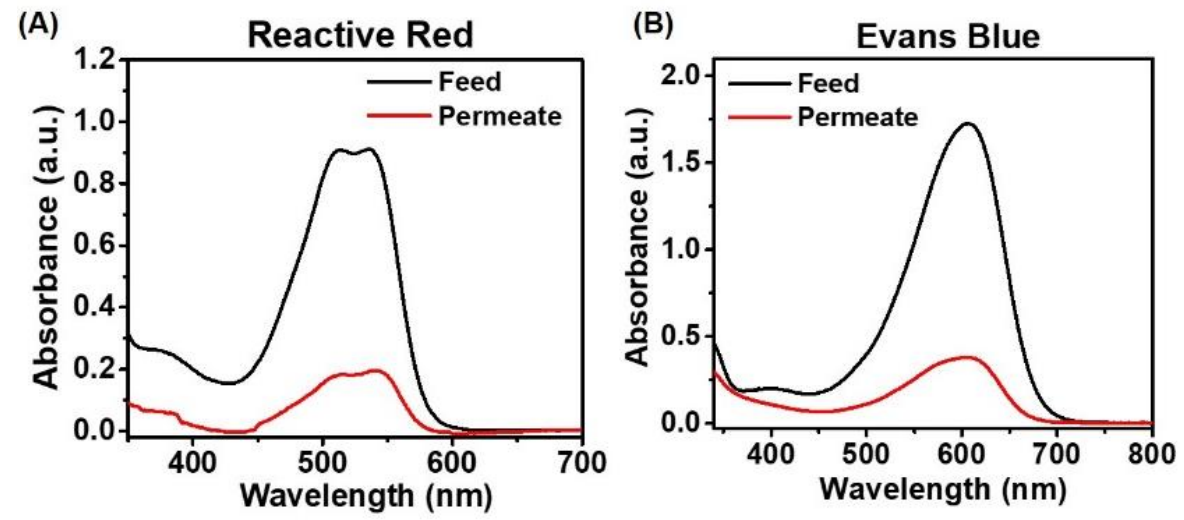

Figure S6. UV-vis absorption spectra of solutions of (A) RR and (B) EB before and after filtration through the E-MSTF $\perp$ AAO membrane. 


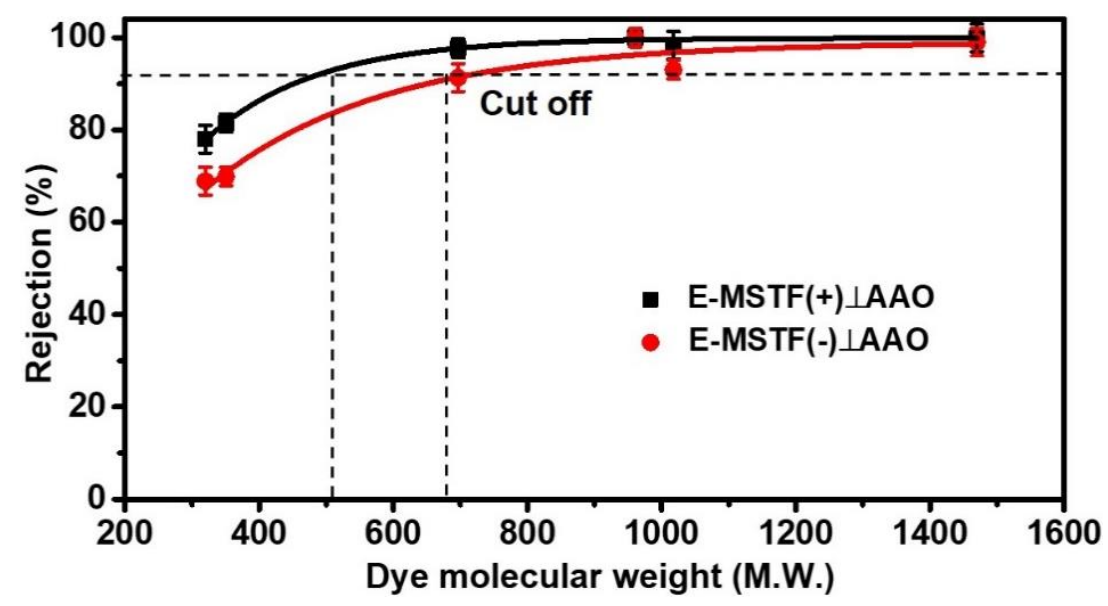

Figure S7. Dye rejection performance of the E-MSTF $(+) \perp A A O$ and E-MSTF $(-) \perp A A O$ membranes.
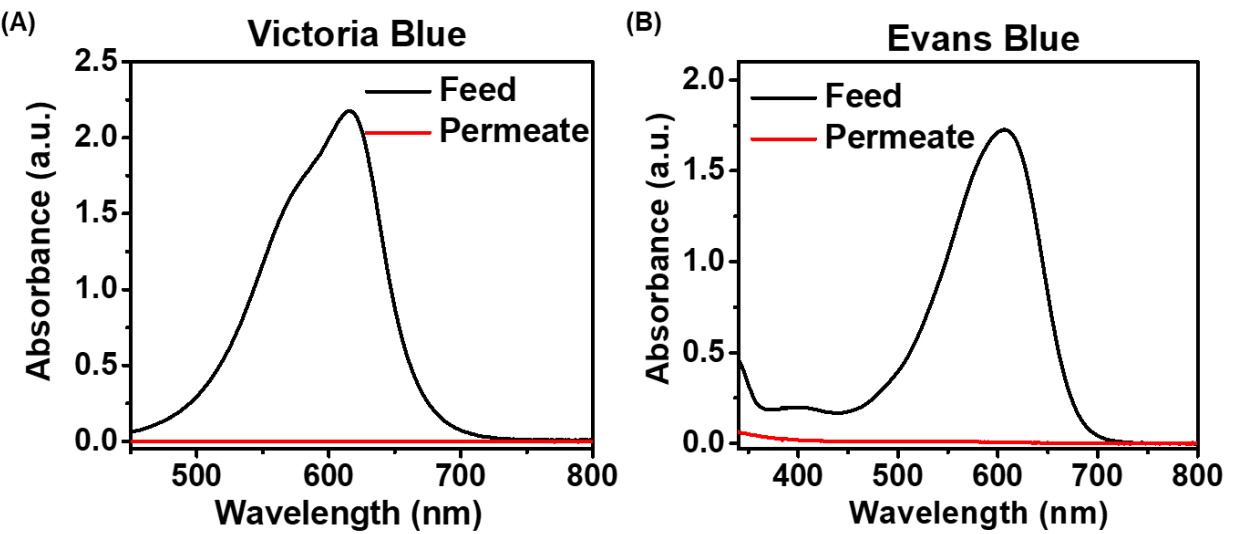

Figure S8. UV-vis spectra of (A) positively charged dye (Victoria blue, $500 \mathrm{ppm}$ ) solution and (B) negatively charged dye (Evans blue, 500 $\mathrm{ppm}$ ) solution before and after filtration test. 


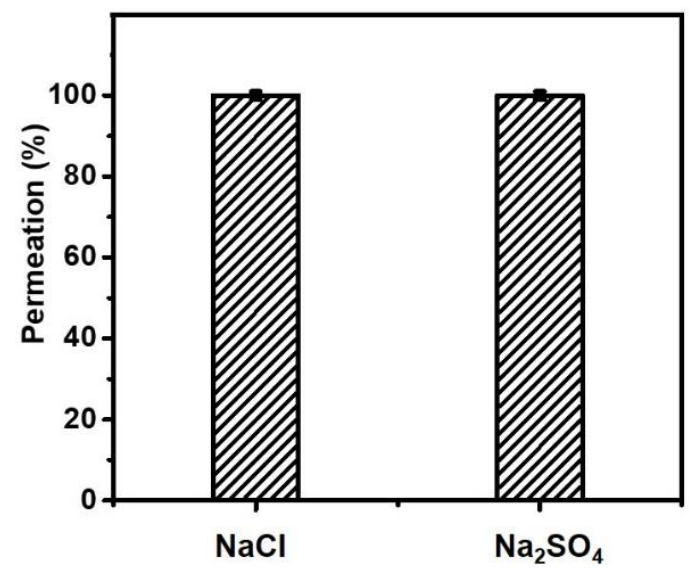

Figure S9. Salt permeation performance of the E-MSTF $(+) \perp \mathrm{AAO}$ membrane.

\section{Reference}

1. J. Yang, G.-S. Lin, C.-Y. Mou and K.-L. Tung, Chem. Mat., 2019, 31, 1745-1751.

2. S. Yu, Y. Zheng, Q. Zhou, S. Shuai, Z. Lü and C. Gao, Desalination, 2012, 298, 49-58.

3. S. Yu, M. Liu, M. Ma, M. Qi, Z. Lü and C. Gao, Journal of Membrane Science, 2010, 350, 83-91.

4. M. Liu, Y. Zheng, S. Shuai, Q. Zhou, S. Yu and C. Gao, Desalination, 2012, 288, 98-107.

5. L. Yu, J. Deng, H. Wang, J. Liu and Y. Zhang, ACS Sustainable Chemistry \& Engineering, 2016, 4, 3292-3304.

6. J. Lin, W. Ye, H. Zeng, H. Yang, J. Shen, S. Darvishmanesh, P. Luis, A. Sotto and B. Van der Bruggen, Journal of Membrane Science, 2015, 477, 183-193.

7. J. Zhu, M. Tian, J. Hou, J. Wang, J. Lin, Y. Zhang, J. Liu and B. Van der Bruggen, Journal of Materials Chemistry A, 2016, 4, 19801990.

8. J. Zhu, A. Uliana, J. Wang, S. Yuan, J. Li, M. Tian, K. Simoens, A. Volodin, J. Lin and K. Bernaerts, Journal of Materials Chemistry A, 2016, 4, 13211-13222.

9. M. Jiang, K. Ye, J. Deng, J. Lin, W. Ye, S. Zhao and B. Van der Bruggen, Environmental Science \& Technology, 2018, 52, 10698-10708. 